\title{
Adubação orgânica no sulco de plantio e sua influência no desenvolvimento do sorgo
}

\section{Organic manuring the furrow its influence the development of sorghum}

\author{
Gilson Araujo de Freitas ${ }^{1}$, Cintia Ribeiro de Sousa ${ }^{1}$, Aristóteles Capone ${ }^{1}$, Flávio Sérgio \\ Afférri $^{1}$, Aurélio Vaz de Melo ${ }^{1}$ e Rubens Ribeiro da Silva ${ }^{1}$ \\ ${ }^{I}$ Departamento de Agronomia; Universidade Federal do Tocantins; 77402-970; Gurupi - TO - Brasil.
}

\begin{abstract}
In the last years the sorghum production in Brazil has increased so much, as consequence of the expansion of the planted area and productivity increments. However, studies of the organic manuring on the development of the culture are still incipient. In that way, it was aimed at to evaluate the effect of the applied organic manuring in the planting furrow in the development of plants of Sorghum bicolor. The experiment was droven in randomized blocks design, with six repetitions, being the hybrid of grain sorghum A9735R submitted to eight treatments: 0, 10, 20, 30, 40, 50 and $60 \mathrm{tha}^{-1}$ of organic fertilizer applied in the planting furrow and $500 \mathrm{~kg} \mathrm{ha}^{-1}$ of the formulation 04-14-08 $+\mathrm{Zn}$. The concentrations of organic manuring in the planting furrow provided differences among the treatments. Being observed that you plant submitted to the doses 40 to 60 t ha-1 organic manuring presented better vegetative acting for the appraised parameters. The largest answer of growth of the stem was what received $40 \mathrm{tha^{-1 }}$ of organic manuring.
\end{abstract}

Key-words: Organic agriculture, agroecology, Sorghum bicolor, sustainability

\section{INTRODUÇÃO}

O sorgo (Sorghum bicolor L. Moench) é uma planta com altas taxas fotossintéticas que pode ser cultivada em quase todo território nacional. Esta cultura é de enorme utilidade em regiões muito quentes e muito secas, onde culturas como o milho, não atinge o máximo em produtividade de grãos ou de forragem (Molina et al, 2000).

Quinto cereal mais cultivado no mundo, é utilizado principalmente na alimentação animal e como matéria-prima para a produção de álcool anidro, bebidas alcoólicas, tintas e vassouras. Porém, a produtividade média de sorgo no Brasil ainda é considerada baixa, girando em torno de $2.276 \mathrm{~kg} \mathrm{ha}^{-1} \mathrm{de}$ grãos. Dentre os principais fatores responsáveis pela baixa produtividade nas áreas destinadas à produção dessas plantas destacam-se as precipitações irregulares, a fertilidade do solo e as baixas aplicações de fertilizantes (Aguiar et al., 2007). Caracteriza-se também como uma cultura que responde à calagem e à adubação com macronutrientes (Alvarez Venegas et al., 1978).

Bulluck et al. (2002), afirmam que compostos orgânicos usados como melhoradores alternativos da fertilidade do solo, podem resultar em incremento da matéria orgânica e atividade biológica do solo visando não só à melhoria das propriedades físicas e químicas do solo (Costa et al., 2001) também à redução das quantidades de adubos químicos que são aplicados para as plantas (Ricci et al., 1995).

O sistema de manejo do solo adequado favorece o desenvolvimento radicular e a absorção de nutrientes e, por conseguinte, o desenvolvimento das plantas (Arf et al., 2002). Dentre esses sistemas, o manejo orgânico tem apresentado diversas vantagens. Para Hoffman (2001), os benefícios no uso de estercos animais podem ser assim elencados: melhorias nas propriedades físicas do solo e no fornecimento de nutrientes;

Author for correspondence: araujoagro@hotmail.com 
aumento no teor de matéria orgânica, melhora na infiltração da água bem como também aumento na capacidade de troca de cátions. Corretamente manejados e utilizados, revertem-se em fornecedores de nutrientes para a produção de alimentos e melhoradores das condições físicas, químicas e biológicas do solo.

A utilização de resíduos orgânicos animais como fonte de nutrientes para diferentes cadeias produtivas de vegetais representa alternativas eficientes que visam à diminuição ou eliminação da dependência dos fertilizantes químicos e aumento da segurança ambiental. Segundo Konzen e Alvarenga (2005) se verificou que a adubação orgânica, utilizando estercos de suínos, aves e bovinos constituem fertilizantes eficientes na produção de milho, tanto para grãos quanto para forragem. De acordo com Malavolta et al. (2002) quimicamente, a adubação orgânica é importante fonte de nutrientes, especialmente $\mathrm{N}, \mathrm{P}, \mathrm{K}$ e micronutrientes, sendo a única forma de armazenamento de $\mathrm{N}$ que não volatiliza e, ainda, responsável por $80 \%$ do fósforo total encontrado no solo.

No entanto, prejuízos aos vegetais, decorrentes da adição de grandes quantidades de adubos orgânicos, podem ser ocasionados por diversos fatores, entre os quais: diminuição no suprimento de oxigênio, estresse hídrico (Guenzi et al., 1978), e presença de quantidades tóxicas de amônia e de sais, principalmente os de potássio (Santos et al., 1981).

Regiões de clima tropical e subtropical apresentam predominância de solos muito intemperizados, com baixos conteúdos de matéria orgânica e nutrientes disponíveis (Brady, 1989). Essas condições são comumente encontradas na região sul de Tocantins, tornando o uso agrícola de resíduos orgânicos, como o esterco de gado, um recurso vantajoso que tem sido recomendado por proporcionar benefícios agronômicos, como elevação do pH do solo (Silva et al., 2001), redução da acidez potencial e aumento na disponibilidade de macronutrientes (Berton et al., 1997), além de representar um benefício de ordem social, pela disposição final menos impactante do resíduo no ambiente.

Assim, objetivou-se com esse trabalho, avaliar o efeito da adubação orgânica aplicada no sulco de plantio no desenvolvimento de plantas de Sorghum bicolor.

\section{MATERIAL E MÉTODOS}

O presente estudo foi conduzido na área experimental da Universidade Federal de Tocantins (UFT), Campus Universitário de Gurupi, localizado na região sul do estado do Tocantins, a $280 \mathrm{~m}$ de altitude, nas coordenadas $11^{\circ} 43^{\prime} 45^{\prime \prime}$ de latitude sul e $49^{\circ} 04^{\prime} 07^{\prime \prime}$ de longitude oeste. Segundo a classificação climática de Köppen (1948), o clima regional é do tipo B1wA'a' úmido com moderada deficiência hídrica. A temperatura média anual é de $29,5^{\circ} \mathrm{C}$, com precipitação média anual de $1804 \mathrm{~mm}$.

$\mathrm{O}$ experimento foi conduzido em delineamento de blocos ao acaso, com seis repetições, sendo o híbrido de sorgo granífero A 9735R submetido a oito tratamentos: $0,10,20,30,40,50$ e $60 \mathrm{t} \mathrm{ha}^{-1} \mathrm{de}$ adubo orgânico aplicados no sulco de plantio e $500 \mathrm{~kg} \mathrm{ha}^{-1}$ da formulação 04-14-08 + Zn também no sulco. A adubação de cobertura foi realizada aos 35 dias após a emergência das plântulas de acordo com a necessidade da cultura e análise do solo. O adubo orgânico (esterco bovino curtido) foi aplicado diretamente no sulco de plantio e levemente incorporado ao solo.

$\mathrm{O}$ solo utilizado no experimento apresentou as seguintes características químicas: $\mathrm{pH}\left(\mathrm{H}_{2} \mathrm{O}\right)-5,2$; Matéria Orgânica - 2,1\% ; Cálcio - 2,1 $\mathrm{cmol}_{\mathrm{c}} \mathrm{dm}^{-}$ 3. Magnésio - 0,4 $\mathrm{cmol}_{\mathrm{c}} \mathrm{dm}^{-3}$; Potássio - 18,0 mg $\mathrm{kg}^{-1}$; Fósforo $-5,0 \mathrm{mg} \mathrm{kg}$. O esterco utilizado foi oriundo da estação de tratamento de resíduos da bovinocultura da COOPERFRIGU (Cooperativa dos Produtores de Carne e Derivados de Gurupi TO) e apresentou: $\mathrm{pH}\left(\mathrm{CaCl}_{2}\right)$ - 6,9; Matéria Orgânica - 7,3\% ; Cálcio - 4,0 $\mathrm{cmol}_{\mathrm{c}} \mathrm{dm}^{-3}$; Magnésio - 2,3 $\mathrm{cmol}_{\mathrm{c}} \mathrm{dm}^{-3}$; Alumínio - $0,0 \mathrm{cmol}_{\mathrm{c}}$ $\mathrm{dm}^{-3}$; Hidrogênio + Alumínio - 0,4 $\mathrm{cmol}_{\mathrm{c}} \mathrm{dm}^{-3}$; Potássio - 103,1 $\mathrm{mg} \mathrm{L}^{-1}$; Fósforo - 683,0 $\mathrm{mg} \mathrm{L}^{-1}$, e Sódio - 33,8 $\mathrm{mg} \mathrm{L}^{-1}$.

O plantio do híbrido de sorgo granífero A 9735R foi em Latossolo Vermelho Amarelo distrófico. Foram distribuídas sementes de sorgo diretamente sobre o esterco, em parcelas de dois metros lineares, com o objetivo de obter uma população de 200.000 plantas por hectare. Foram distribuídas 160 sementes de sorgo por parcela de dois metros de comprimento com espaçamento de um metro entre linhas e entre plantas de cinco centímetros.

O desbaste foi efetuado aos sete dias após a emergência das plântulas deixando-se o equivalente a 20 plantas por metro linear. Logo após, foram iniciadas as avaliações de altura, diâmetro e número de folhas. As análises foram realizadas semanalmente durante o ciclo da cultura 
até a colheita e ao final foram coletadas as outras variáveis.

Os parâmetros avaliados foram: Altura de planta adulta, Altura de Plantas com Cacho (APCC), Altura de Plantas sem Cacho (APSC), Diâmetro do Colmo (DC), Diâmetro Médio do Colmo (DMC), Diâmetro da Panícula (DP), Número de Folhas (NF), Área Foliar (AF), Massa Verde da Planta (MVP), Peso da Panícula (PP), Comprimento da Panícula (CP), Numero de Espiguetas (NE).

Para avaliação de altura de planta, diâmetro do colo e número de folhas foram avaliadas dez plantas em cada parcela semanalmente durante todo ciclo da cultura. A altura da planta foi considerada desde o colo até o ápice de cada planta e para o diâmetro do colo foi tomado a $3 \mathrm{~cm}$ do solo. Para as avaliações das outras variáveis, os dados foram obtidos ao final do experimento. No caso da produção de espiguetas, foram avaliadas em cinco plantas por parcela. Não foi realizar a colheita do sorgo, devido à alta população de maritacas, que consumiu praticamente toda produção de grãos.

Os parâmetros avaliados foram analisados estatisticamente, com base nas recomendações encontradas em Gomes (1990). As médias foram comparadas através do teste de Tukey a 5\% de probabilidade.

\section{RESULTADOS E DISCUSSÕES}

No estádio inicial de crescimento das plantas de sorgo, observou-se que o tratamento que recebeu a dose de 50 t ha- ${ }^{1}$ de adubação orgânica no sulco de plantio e adubação química obtiveram melhores desempenhos para a característica altura de planta, diferenciando-se estatisticamente dos outros tratamentos ( $a=9,47$ e $a=9,25$, respectivamente). Entretanto, ao longo do período de avaliação, observou-se que as respostas das plantas de sorgo submetidas ao tratamento com adubação química não se manteve. Essas apresentaram menor velocidade de crescimento $(b=2,41)$, não diferindo, por exemplo, controle $(b=2,51)$. A dose que proporcionou maior resposta na velocidade de crescimento das plantas de sorgo foi a de $60 \mathrm{t} \mathrm{ha}^{-1}$ de adubação orgânica $(b=2,71)$ diferenciando-se estatisticamente dos demais tratamentos com exceção da dose $40 \mathrm{t} \mathrm{ha}^{-1}$ de adubação orgânica $(b=2,60)$ (Tabela 1).

Considerando os resultados obtidos com a adubação química pode-se inferir que, diferentemente da adubação orgânica, esta poderia ter disponibilizado os nutrientes para as plantas de forma mais imediata, proporcionando maior arranque no início do crescimento das plantas. Já os tratamentos submetidos à adubação orgânica responderam de forma mais gradual ao longo do ciclo vegetativo da cultura, que pode ter ocorrido em função da liberação dos nutrientes não ser tão rápida quanto na adubação química. Entretanto, podem suprir as carências nutricionais das plantas por um período de tempo maior do que a adubação química.

Tabela 1. Altura de planta, diâmetro de colmo e número de folhas de plantas de sorgo granífero (Sorghum bicolor) aos trinta dias após emergência submetidos a concentrações de adubação orgânica (A.O.) no sulco de plantio no município de Gurupi - TO.

\begin{tabular}{cccccccccc}
\hline & \multicolumn{3}{c}{ Altura de planta $(\mathrm{cm})$} & \multicolumn{3}{c}{ Diâmetro de colmo $(\mathrm{mm})$} & \multicolumn{3}{c}{ Número de folhas } \\
Tratamentos & \multicolumn{3}{c}{$\mathrm{y}=\mathrm{a}+\mathrm{bx}$} & \multicolumn{3}{c}{$\mathrm{y}=\mathrm{a}+\mathrm{bx}$} & \multicolumn{3}{c}{$\mathrm{y}=\mathrm{a}+\mathrm{bx}$} \\
\cline { 2 - 10 } & $\mathrm{a}$ & $\mathrm{b}$ & $\mathrm{r}^{2}$ & $\mathrm{a}$ & $\mathrm{b}$ & $\mathrm{r}^{2}$ & $\mathrm{a}$ & $\mathrm{b}$ & $\mathrm{r}^{2}$ \\
\hline $0 \mathrm{t} \mathrm{ha}^{-1}$ A.O. & $-1,38 \mathrm{e}$ & $2,51 \mathrm{ab}$ & $0,995 \mathrm{a}$ & $2,59 \mathrm{~b}$ & $0,31 \mathrm{abc}$ & $0,936 \mathrm{a}$ & $4,93 \mathrm{~cd}$ & $0,13 \mathrm{a}$ & $0,997^{\mathrm{a}}$ \\
$10 \mathrm{t} \mathrm{ha}^{-1}$ A.O. & $-0,42 \mathrm{de}$ & $2,50 \mathrm{ab}$ & $0,993 \mathrm{~b}$ & $2,49 \mathrm{ab}$ & $0,31 \mathrm{c}$ & $0,930 \mathrm{a}$ & $5,09 \mathrm{~cd}$ & $0,12 \mathrm{a}$ & $0,985^{\mathrm{a}}$ \\
$20 \mathrm{t} \mathrm{ha}^{-1}$ A.O. & $2,88 \mathrm{cde}$ & $2,47 \mathrm{~b}$ & $0,995 \mathrm{a}$ & $3,39 \mathrm{ab}$ & $0,29 \mathrm{c}$ & $0,897 \mathrm{a}$ & $4,92 \mathrm{~d}$ & $0,13 \mathrm{a}$ & $0,980^{\mathrm{a}}$ \\
$30 \mathrm{t} \mathrm{ha}^{-1}$ A.O. & $4,07 \mathrm{bcd}$ & $2,54 \mathrm{ab}$ & $0,99 \mathrm{ab}$ & $3,12 \mathrm{ab}$ & $0,31 \mathrm{bc}$ & $0,913 \mathrm{a}$ & $5,03 \mathrm{bcd}$ & $0,13 \mathrm{a}$ & $0,980^{\mathrm{a}}$ \\
$40 \mathrm{t} \mathrm{ha}^{-1}$ A.O. & $5,47 \mathrm{abc}$ & $2,6^{\mathrm{a}}$ & $0,99 \mathrm{ab}$ & $3,18 \mathrm{ab}$ & $0,35^{\mathrm{a}}$ & $0,897 \mathrm{a}$ & $5,38 \mathrm{ab}$ & $0,13 \mathrm{a}$ & $0,974^{\mathrm{a}}$ \\
$50 \mathrm{t} \mathrm{ha}^{-1}$ A.O. & $9,47^{\mathrm{a}}$ & $2,46 \mathrm{~b}$ & $0,985 \mathrm{ab}$ & $3,63 \mathrm{a}$ & $0,34 \mathrm{ab}$ & $0,849 \mathrm{a}$ & $5,51 \mathrm{ab}$ & $0,13 \mathrm{a}$ & $0,993 \mathrm{a}$ \\
$60 \mathrm{t} \mathrm{ha}^{-1}$ A.O. & $0,37 \mathrm{abc}$ & $2,71^{\mathrm{a}}$ & $0,885 \mathrm{~b}$ & $3,57 \mathrm{ab}$ & $0,33 \mathrm{bc}$ & $0,883 \mathrm{a}$ & $5,72 \mathrm{a}$ & $0,12 \mathrm{a}$ & $0,978 \mathrm{a}$ \\
$500 \mathrm{k} \mathrm{ha}^{-1}$ NPK & $9,25 \mathrm{ab}$ & $2,41 \mathrm{~b}$ & $0,992 \mathrm{a}$ & $4,18 \mathrm{a}$ & $0,29 \mathrm{bc}$ & $0,907 \mathrm{a}$ & $5,33 \mathrm{abc}$ & $0,12 \mathrm{a}$ & $0,965 \mathrm{a}$ \\
\hline
\end{tabular}

Média seguidas pela mesma letra nas colunas não diferem estatisticamente ao nível de probabilidade de 5\% pelo teste de Tukey. 
Para Rolas (1994), os nutrientes presentes no adubo orgânico também podem ser disponibilizados de forma imediata à planta, contribuindo para o seu desenvolvimento, uma vez que parte do $\mathrm{N}$ contido nesses materiais está na forma mineral, comportando-se semelhantemente ao $\mathrm{N}$ contido nos fertilizantes minerais, concordando com os resultados aqui encontrados. Ainda reforça que todo o potássio aplicado com o adubo orgânico comporta-se como mineral desde a aplicação e não precisa sofrer a ação dos microorganismos.

Além disso, segundo Malavolta et al., (1997), a utilização de matéria orgânica no solo promove mudanças nas suas características físicas, químicas e biológicas, pois melhora a estrutura do solo, aumenta a capacidade de retenção de água e a aeração, permitindo maior penetração e distribuição das raízes. Pois, quimicamente, a matéria orgânica é a principal fonte de macro e micronutrientes, que são importantes no desenvolvimento e produção das plantas.

Com relação ao diâmetro do colmo, foi observado que os tratamentos influenciaram de forma semelhante à característica altura de planta (Tabela 1), sendo que, neste caso, o tratamento que proporcionou maior resposta de crescimento do colmo foi o que recebeu $40 \mathrm{t} \mathrm{ha}^{-1}$ de adubação orgânica $(b=0,35, p<=0,05)$.

Considerando que não houve restrição na disponibilidade de agua no solo, a baixa capacidade da adubação química em manter o solo úmido que pode ter afetado a absorção de água pelas plantas submetidas a esse tratamento, levando a perda de turgescência celular, o que deve ter promovido o fechamento estomático, redução na assimilação de $\mathrm{CO}_{2}$ e inibição do processo fotossintético. (Larcher, 1986; Taiz e Zeiger, 1998) afetando, assim, o crescimento do colmo, uma vez que este tem papel importante no acúmulo de reservas da planta.
Desta forma, seja pela absorção de nutrientes importantes ou por proporcionar um microclima no solo favorável ao desenvolvimento cultura, a adubação orgânica se mostra eficiente quando aplicada no sulco de plantio e principalmente nas doses de 40, 50 e $60 \mathrm{t}$ ha-1, tanto para a altura quanto para o diâmetro de colmo das plantas. Silva et al., (2005), afirma que essas características estão relacionadas ao rendimento sorgo e Zago (1992) ressalta que os sorgos mais altos apresentam maiores rendimentos de matéria seca. Aspectos como germinação, altura de plantas, diâmetro do colmo, e ângulo foliar são características essenciais utilizadas no intuito de selecionar variedades ou cultivares adaptadas a cada região, assegurando, uma boa produtividade.

As doses de adubação orgânica aplicadas no sulco de plantio proporcionaram diferenças estatísticas apenas nas avaliações iniciais, quando o número de folhas emitidas pelas plantas foi maior nos tratamentos que receberam adubação química e 40, 50 e $60 \mathrm{t} \mathrm{ha}^{-1}$ de adubação orgânica ( $\mathrm{a}=5,33 ; 5,38$; 5,51 e 5,72, respectivamente). Desta forma, os resultados se mostram semelhantes aos encontrados para as características altura de planta e diâmetro do colmo, com exceção da velocidade de respostas aos tratamentos, onde não houve diferenças significativas (Tabela 1).

Foi observado que houve diferença significativa para a característica altura de planta com cacho (APCC). Os tratamentos que proporcionaram as maiores APCC foram 40, 50 e $60 \mathrm{t} \mathrm{ha}^{-1}$ de adubo orgânico no sulco (Tabela 2). Enquanto as menores alturas foram observadas nos tratamentos com doses 0 e $10 \mathrm{t} \mathrm{ha}^{-1}$ de adubo orgânico no sulco. Comparando altura de planta com cacho (APCC) e altura de planta sem cacho (APSC) verifica-se que as diferenças significativas ocorreram em função do tamanho do cacho, isso porque a APCC é a soma de APSC com tamanho do cacho (TC), que separados não mostraram diferenças significativas. 
Tabela 2. Altura da planta com cacho (APCC), altura da planta sem cacho (APSC), tamanho do cacho (TC), diâmetro de panícula (DP), peso de panícula (PP), e número de espiguetas (NE) de sorgo granífero submetidos a diferentes concentrações de adubação orgânica (A.O.) no sulco de plantio no município de Gurupi - TO.

\begin{tabular}{ccccccc}
\hline Tratamentos & APCC $(\mathrm{cm})$ & APSC $(\mathrm{cm})$ & TC & DP & PP & NE \\
\hline $0 \mathrm{t} \mathrm{ha}-^{-1}$ A.O. & $143 \mathrm{~b}$ & $110 \mathrm{a}$ & $33,5 \mathrm{a}$ & $41,6 \mathrm{a}$ & $17,6 \mathrm{a}$ & $59,5 \mathrm{a}$ \\
$10 \mathrm{t} \mathrm{ha}^{-1}$ A.O. & $141 \mathrm{ab}$ & $108 \mathrm{a}$ & $33,1 \mathrm{a}$ & $42,8 \mathrm{a}$ & $17,6 \mathrm{a}$ & $60,5 \mathrm{a}$ \\
$20 \mathrm{t} \mathrm{ha}^{-1}$ A.O. & $145 \mathrm{ab}$ & $109 \mathrm{a}$ & $35,6 \mathrm{a}$ & $43,6 \mathrm{a}$ & $18,0 \mathrm{a}$ & $58,5 \mathrm{a}$ \\
$30 \mathrm{t} \mathrm{ha}^{-1}$ A.O. & $145 \mathrm{ab}$ & $111 \mathrm{a}$ & $34,1 \mathrm{a}$ & $44,7 \mathrm{a}$ & $18,3 \mathrm{a}$ & $60,3 \mathrm{a}$ \\
$40 \mathrm{t} \mathrm{ha}^{-1}$ A.O. & $146 \mathrm{a}$ & $112, \mathrm{a}$ & $34,5 \mathrm{a}$ & $46,8 \mathrm{a}$ & $19,3 \mathrm{a}$ & $61,5 \mathrm{a}$ \\
$50 \mathrm{t} \mathrm{ha}^{-1}$ A.O. & $147 \mathrm{a}$ & $112 \mathrm{a}$ & $34,6 \mathrm{a}$ & $43,7 \mathrm{a}$ & $18,1 \mathrm{a}$ & $60,8 \mathrm{a}$ \\
$60 \mathrm{t} \mathrm{ha}^{-1}$ A.O. & $146 \mathrm{a}$ & $111 \mathrm{a}$ & $34,8 \mathrm{a}$ & $47,7 \mathrm{a}$ & $20,5 \mathrm{a}$ & $58,5 \mathrm{a}$ \\
$500 \mathrm{~kg}^{-1} \mathrm{NPK}$ & $146 \mathrm{ab}$ & $111 \mathrm{a}$ & $34,8 \mathrm{a}$ & $43,2 \mathrm{a}$ & $19,8 \mathrm{a}$ & $61,0 \mathrm{a}$ \\
\hline MÉDIA & 145 & 110 & 34,4 & 44,1 & 18,8 & 60,0 \\
CV $(\%)$ & 1,75 & 3,03 & 6,12 & 9,98 & 14,6 & 5,45 \\
\hline
\end{tabular}

Média seguidas pela mesma letra nas colunas não se diferem estatisticamente ao nível de probabilidade de 5\% pelo teste de Tukey

Apesar de não ter ocorrido diferenças significativas entre os tratamentos, foi observado que as doses 0 e $10 \mathrm{t} \mathrm{ha}^{-1}$ de adubo orgânico no sulco, apresentaram os menores valores para os parâmetros Altura de Plantas com Cacho, Diâmetro do Colmo, Diâmetro da Panícula e Peso da Panícula . Desta forma pressupõe que as doses de até $10 \mathrm{t}$ ha-1 de adubação orgânica não são suficientes para que a planta tenha um bom desenvolvimento dessas características. Fisiologicamente a planta pode ter acumulado menos reserva, resultando em menor altura e menor translocação de fotoassimilados aos órgãos reprodutivos. Os nutrientes têm funções essenciais e específicas no metabolismo das plantas. Dessa forma, quando um dos nutrientes essenciais não está presente em quantidades satisfatórias ou em condições que o tornem pouco disponível, a sua deficiência nas células promove alterações no seu metabolismo, reduzindo o desenvolvimento das plantas (Taiz e Zeiger, 2004).

Com relação ao Diâmetro Inferior do Colmo (DIC), Diâmetro Médio do Colmo (DMC), Área Foliar (AF) e Massa Verde da Planta (MVP), houve tendência das plantas submetidas à dose de 40 t ha- $^{1}$ de adubo orgânico no sulco, serem superiores aos demais tratamentos, e aquelas submetidas às doses de 0 e $10 \mathrm{t} \mathrm{ha}^{-1}$ serem inferiores (Tabela 3). Apesar dessas diferenças, os dados não foram estatisticamente significativos, com exceção do DMC, em que as plantas submetidas a dose de 40t ha ${ }^{-1}$ obtiveram maiores valores para essa característica. A dose $40 \mathrm{t} \mathrm{ha}^{-1} \mathrm{de}$ adubação orgânica proporcionou maior área foliar das plantas, e, embora essa diferença não tenha sido significativa estatisticamente, foi suficiente para influenciar o diâmetro do colmo, pois de acordo com Taiz e Zeiger (1998) sendo a folha a parte da planta de maior taxa fotossinteticamente ativa, esta pode conferir maior diâmetro de caule devido à maior quantidade de fotoassimilados acumulados durante o ciclo, resultando então numa maior quantidade de massa verde.

Alvim et al, (2003) relatam que além da necessidade da planta ser adaptada as condições climáticas da região o seu potencial forrageiro é maximizado quando a fertilidade do solo atende as suas exigências, neste sentido a adubação orgânica é essencial, para garantir o crescimento das plantas, pois é uma adubação completa em termos nutricionais de praticamente todos os elementos que a planta necessita. 
Tabela 3. Diâmetro Inferior do Colmo (DIC), Diâmetro Médio do Colmo (DMC), Área foliar (AF), e Massa Verde da Planta (MVP) de sorgo granífero submetidos a diferentes níveis de adubação orgânica (A.O.) no sulco de plantio no município de Gurupi - TO.

\begin{tabular}{ccccc}
\hline Tratamentos & DIC $(\mathrm{mm})$ & DMC $(\mathrm{mm})$ & AF $(\mathrm{mm} 2)$ & MVP $\left(\mathrm{t} \mathrm{ha}^{-1}\right)$ \\
\hline 0 t ha-1 A.O. & $14,44 \mathrm{a}$ & $14,5 \mathrm{ab}$ & $502,969 \mathrm{a}$ & $20,620 \mathrm{a}$ \\
$10 \mathrm{t} \mathrm{ha}^{-1}$ A.O. & $14,02 \mathrm{a}$ & $13,83 \mathrm{~b}$ & $471,597 \mathrm{a}$ & $20,380 \mathrm{a}$ \\
$20 \mathrm{t} \mathrm{ha}^{-1}$ A.O. & $14,05 \mathrm{a}$ & $14,66 \mathrm{ab}$ & $489,325 \mathrm{a}$ & $20,653 \mathrm{a}$ \\
$30 \mathrm{t} \mathrm{ha}^{-1}$ A.O. & $14,89 \mathrm{a}$ & $15,16 \mathrm{ab}$ & $490,571 \mathrm{a}$ & $21,593 \mathrm{a}$ \\
$40 \mathrm{t} \mathrm{ha}^{1}$ A.O. & $16,1 \mathrm{a}$ & $16 \mathrm{a}$ & $526,168 \mathrm{a}$ & $23,020 \mathrm{a}$ \\
$50 \mathrm{t} \mathrm{ha}^{-1}$ A.O. & $14,91 \mathrm{a}$ & $15,33 \mathrm{ab}$ & $503,119 \mathrm{a}$ & $22,086 \mathrm{a}$ \\
$60 \mathrm{t} \mathrm{ha}^{-1}$ A.O. & $15,34 \mathrm{a}$ & $16,16 \mathrm{a}$ & $489,080 \mathrm{a}$ & $22,373 \mathrm{a}$ \\
500 kg ha-1 $^{1}$ NPK & $14,61 \mathrm{a}$ & $14,66 \mathrm{ab}$ & $492,239 \mathrm{a}$ & $23,026 \mathrm{a}$ \\
\hline MÉDIA & 14,79 & 15,04 & 495,633 & 21,719 \\
CV(\%) & 7,71 & 7,65 & 7,41 & 10,344 \\
\hline
\end{tabular}

Médias seguidas pela mesma letra nas colunas não se diferem estatisticamente ao nível de probabilidade $5 \%$ pelo teste de Tukey.

Desde que a estrutura de uma folhagem pode ser um importante fator para determinar a produtividade de uma comunidade vegetal, a avaliação cuidadosa da área foliar é sem dúvida fator que auxilia na tomada de decisão para se eleger uma cultivar mais produtiva (Magalhães, 1979). O significado deste parâmetro resume-se na premissa que materiais mais produtivos, possuem uma maior facilidade em manter uma área foliar por um maior período, possibilitando um melhor desempenho do aparato fotossintético.

\section{CONCLUSÃO}

Mesmo em doses alevadas de adubo orgânico, não foi observado fitotoxidez nas plantas de sorgo granifero. Ao contrario, se mostra eficiente quando aplicada no sulco de plantio nas doses de 40, 50 e $60 \mathrm{t} \mathrm{ha}^{-1}$.

\section{RESUMO}

Nos últimos anos a produção de sorgo no Brasil cresceu tanto, como consequência da expansão da área plantada e incrementos de produtividade. No entanto, os estudos sobre a adubação orgânica sobre o desenvolvimento da cultura ainda são incipientes. Desta forma, foi destinado a avaliar o efeito da adubação aplicada orgânico no sulco no desenvolvimento de plantas de sorgo. O experimento foi droven em delineamento de blocos casualizados, com seis repetições, sendo o híbrido de sorgo A9735R submetidos a oito tratamentos: 0, 10, 20, 30, 40, 50 e 60 t ha- $^{1}$ de fertilizante orgânico aplicado no sulco de plantio e $500 \mathrm{~kg}$ ha-1 da formulação 04-14$08+\mathrm{Zn}$. As concentrações de adubação orgânica no sulco de plantio apresentou diferenças entre os tratamentos. Sendo observado que você planta apresentada às doses de 40-60 t ha-1 adubação orgânica apresentou melhor atuação vegetativo para os parâmetros avaliados. A maior resposta de crescimento do caule foi o que recebeu $40 \mathrm{t} \mathrm{ha}^{-1}$ de adubação orgânica.

Palavras-chave: Agricultura orgânica, agroecologia, Sorghum bicolor, sustentabilidade

\section{REFERENCIAS}

Alvarez Venegas, V. H.; Defelipo, B. V.; Barros, N. F. de. (1978), Resposta do sorgo à aplicação de micronutrientes num Latossolo VermelhoAmarelo de Itamarandiba, Minas Gerais. Ceres, 25,79-86.

Alvim, M. J.; Brotel, M. A.; Rezende, H.; Xavier, D. F. (2003), Avaliação sob pastejo do potencial forrageiro de gramíneas do gênero Cynodon, sob níveis de nitrogênio e potássio. Revista Brasileira de Zootecnia, Viçosa, 32, 47-54.

Aguiar L. M. S; Moraes, A. V. de C. de; Guimarães, D. P. (2011), Cultivo do sorgo. Embrapa Milho e Sorgo. Sistemas de Produção, 2 Versão Eletrônica -3 Ed. Disponível em: http:// www.cnpms.embrapa.br/publicacoes/sorgo/clima. htm. Acesso em: 20 nov. 2011.

Arf, O.; Rodrigues, R. A. F.; Sá, M. E.; Crusciol, C. A. C.; Pereira J. C. R. (2002), Preparo do solo, irrigação por aspersão e rendimento de engenho do arroz de terras altas. Scientia Agrícola, 59, 321-326.

Berton, R. S.; Valadares, J. M. A. S.; Camargo, O. A.; Bataglia, O. C. (1997), Peletização do lodo de esgoto e adição de $\mathrm{C}_{\mathrm{a}} \mathrm{CO}_{3}$ na produção de matéria seca e absorção de $\mathrm{Zn}$, Cu e Ni pelo milho em três 
latossolos. Revista Brasileira de Ciência do Solo, 21, 685-691.

Brady, N. C. (1989), Natureza e propriedades do solo. 7 Ed., Rio de Janeiro: Freitas Bastos, 878p.

Bulluck, L. R.; Brosius, M.G.; Evanylo, K.; Ristaino, J. B. (2002), Organic and synthetic fertility amendments influence soil microbial, physical and chemical properties on organic and conventional farms. Applled Soll Ecology, 19, 147160.

Costa, C. A.; Casali, V. W. D.; Loures, E. G.; Cecon, P. R.; Jordão, C. P. (2001),Teor de metais pesados em alface (Lactuca sativa L.) adubada com composto orgânico de lixo urbano. Horticultura Brasileira, 19, 10-16.

Gomes, F. P. (1990), Curso de estatística experimental. 13 Ed., Piracicaba: Nobel, 468p.

Guenzi, W. D.; Beard, W. E.; Watanabe, F. S.; Olsen, S. R; Porter, L. K. (1978), Nitrification and denitrification in cattle manure-amended soil. Journal Environment Quality, 7, 196-202.

Larcher, W. (1986), Ecofisiologia vegetal. São Paulo: EPU, 319p.

Magalhães, A. C. N. (1979), Análise quantitativa do crescimento. In: Ferri, M. G. Fisiologia Vegetal. EPU/EDUSP, São Paulo. 1, 331-350.

Malavolta, E.; Vitti, G. C.; Oliveira, S. A. (1997), Avaliação do estado nutricional das plantas: Princípios e aplicações. 2 Ed., Piracicaba: Potafos, 201p.

Malavolta, E.; Pimentel-Gomes, F.; Alcarde, J. C. (2002), Adubos e adubações. São Paulo: Nobel, 200p.

Molina, L. R.; Gonçalves, L. C.; Rodriguez, N. M.; Rodrigues, J. A. S.; Ferreira, J. J.; Ferreira, V. C. P. (2000), Avaliação agronômica de seis híbridos de sorgo (Sorghum bicolor (L.) Moench). Arquivo Brasileiro de Medicina Veterinária e Zootecnia, 52, 385-390.

Ribas, P. M. (2003), Sorgo: Introdução e importância econômica. Sete Lagoas: Embrapa
Milho e Sorgo (Embrapa Milho e Sorgo. Documentos, 26) 16p.

Konzen, E. A. e Alvarenga, R. A. (2005), Manejo e Utilização de Dejetos Animais: Aspectos agronômicos e ambientais. Ministério da Agricultura, Pecuária e Abastecimento. Sete Lagoas: Circular Técnica 63, 65p.

Ricci, M. S. F.; Casali, V. W. D.; Cardoso, A. A.; Ruiz, H. A. (1995), Teores de nutrientes em duas cultivares de alface adubadas com composto orgânico. Pesquisa Agropecuária Brasileira, 30, 1035-1039.

Rolas. (1994), Recomendação de adubação e de calagem para os Estados do Rio Grande do Sul e de Santa Catarina. 3 Ed., Passo Fundo, SBCS Núcleo Regional Sul. 224p.

Santos, G. A.; Rossiello, R. O. P.; Fernandes, M. S.; O’Grady, P. C. (1981), Efeitos da vinhaça sobre o pH do solo, a germinação e o acúmulo de potássio em milho. Pesquisa Agropecuária Brasileira, 16, 480-494.

Silva, A. G.; Rocha, V. S.; Cecon, P. R.; Portugal, A. F.; Pina Filho, O. C. (2005), Avaliação dos caracteres agronômicos de cultivares de sorgo forrageiro sob diferentes condições termofotoperiódicas. Revista Brasileira de Milho e Sorgo, 4, 28-44.

Silva, F. C.; Boaretto, A. E.; Berton, R. S.; Zotellih.B.; Pexe, C. A.; Bernardes, E. M. (2001), Efeito do lodo de esgoto na fertilidade de um Argissolo Vermelho-Amarelo cultivado com canade-açúcar. Pesquisa Agropecuária Brasileira, 36, 831-840.

Taiz, L. e Zeiger, E. (1998), Plant physiology. 2 Ed., Sunderland: Sinauer, 792p.

Zago, C. P. (1992), Utilização de sorgo na alimentação de ruminantes. In: EMBRAPA. Centro Nacional de Pesquisa de Milho e Sorgo. Manejo cultural do sorgo para forragem. Sete Lagoas, 9-302. 